\title{
Aspergillus Galactomannan Antigen Test
}

National Cancer Institute

\section{Source}

National Cancer Institute. Aspergillus Galactomannan Antigen Test. NCI Thesaurus. Code C85875.

An enzyme-linked serum immunoassay used for the detection of aspergillosis. 